\title{
Erratum to: Should All Children Admitted with Community Acquired Pneumonia have Blood Cultures Taken?
}

\author{
Eng Meng Lai • Anna Marie Nathan • \\ Jessie A. de Bruyne • Lee Lee Chan
}

Published online: 3 February 2015

(C) Dr. K C Chaudhuri Foundation 2015

\section{Erratum to: Indian J Pediatr}

DOI 10.1007/s12098-014-1565-6

In the original article, acknowledgement to a grant was missed. Below is the acknowledgement section.

This study was partly funded by the UMR grant (no. UM.TNC2/RC/HTM/RP026-14HTM) awarded to AMN and JAdeB by University Malaya. The funders had no role in study design, data collection and analysis, decision to publish, or preparation of the manuscript.

The online version of the original article can be found at http//dx.doi.org/ 10.1007/s12098-014-1565-6.

E. M. Lai $\cdot$ A. M. Nathan $(\bowtie) \cdot$ J. A. de Bruyne

Department of Pediatrics, University Malaya,

50603 Kuala Lumpur, Malaysia

e-mail: psr9900@hotmail.com

A. M. Nathan · J. A. de Bruyne

University Malaya Pediatrics and Child Health Research Group,

University Malaya, 50603 Kuala Lumpur, Malaysia

L. L. Chan

Department of Pediatrics, Sime Darby Medical Centre, 1 Jalan SS

12/1A, Subang Jaya, 47500 Selangor Darul Ehsan, Malaysia 